Central Manifolds, Normal Forms

Peer-reviewed author version

BONCKAERT, Patrick (2006) Central Manifolds, Normal Forms. In: Francoise, Jean-Pierre \& Naber, Gregory L. \& Tsun, T.S. (Ed.) Encyclopedia of mathematical physics, vol: $1,2,3,4,5$, p. $467-472$.

DOI: 10.1016/B0-12-512666-2/00092-4

Handle: http://hdl.handle.net/1942/1561 


\title{
Central manifolds, normal forms
}

\author{
Patrick Bonckaert \\ Hasselt University \\ Agoralaan, gebouw D \\ B-3590 Diepenbeek, Belgium
}

September 5, 2005

\section{Introduction}

We consider differentiable dynamical systems generated by a diffeomorphism or a vector field on a manifold. We restrict to the finite-dimensional case, although some of the ideas can also be developed in the general case [21]. We also restrict to the behavior near a stationary point or a periodic orbit of a flow.

Let the origin 0 of $\mathbf{R}^{n}$ be a stationary point of a $C^{1}$ vector field $X$, i.e. $X(0)=0$. We consider the linear approximation $A=d X(0)$ of $X$ at 0 and its spectrum $\sigma(A)$, which we decompose as $\sigma(A)=\sigma_{s} \cup \sigma_{c} \cup \sigma_{u}$ where $\sigma_{s}$ resp. $\sigma_{c}$ resp. $\sigma_{u}$ consists of those eigenvalues with real part $<0$ resp. $=0$ resp. $>0$. If $\sigma_{c}=\emptyset$ then there is no central manifold, and the stationary point 0 is called hyperbolic. Let $E_{s}, E_{c}$ and $E_{u}$ be the linear $A$-invariant subspaces 
corresponding to $\sigma_{s}$ resp. $\sigma_{c}$ resp. $\sigma_{u}$. Then $\mathbf{R}^{n}=E_{s} \oplus E_{c} \oplus E_{u}$. We look for corresponding $X$-invariant manifolds in the neighbourhood of 0 , in the form of graphs of maps. More precisely:

Theorem 1 Let the vector field $X$ above be of class $C^{r}(1 \leq r<\infty)$. There exist map germs $\phi_{s s}:\left(E_{s}, 0\right) \rightarrow E_{c} \oplus E_{u}, \phi_{s c}:\left(E_{s} \oplus E_{c}, 0\right) \rightarrow E_{u}, \phi_{u u}:$ $\left(E_{u}, 0\right) \rightarrow E_{s} \oplus E_{c}, \phi_{c u}:\left(E_{c} \oplus E_{u}, 0\right) \rightarrow E_{s}$ and $\phi_{c}:\left(E_{c}, 0\right) \rightarrow E_{s} \oplus E_{u}$ of class $C^{r}$ such that the graphs of these maps are invariant for the flow of X. Moreover these maps are of class $C^{r}$, and their linear approximation at 0 is zero, i.e. their graphs are tangent to respectively $E_{s}, E_{s} \oplus E_{c}, E_{u}, E_{c} \oplus E_{u}$ and $E_{c}$. If $X$ is of class $C^{\infty}$ then $\phi_{s s}$ and $\phi_{u u}$ are also of class $C^{\infty}$. If $X$ is analytic then $\phi_{s s}$ and $\phi_{u u}$ are also analytic.

The graph of $\phi_{c}$ is called the (local) central (or: centre, center) manifold of $X$ at 0 and it is often denoted by $W^{c}$. Thus: it is an invariant manifold of $X$ tangent at the generalized eigenspace of $d X(0)$ corresponding to the eigenvalues having zero real part.

\section{2 (Non-) uniqueness, smoothness}

Most proofs in the literature [20] use a cut-off in order to construct globally defined objects, and then obtain the invariant graph as the solution of some fixed point problem of a contraction in an appropriate function space. Although this solution is unique for the globalized problem, this is not the case at the germ level: another cut-off may produce a different germ of a central manifold. In 
other words: locally a central manifold might not be unique, as is easily seen on the planar example $x^{2} \partial / \partial x-y \partial / \partial y$. On the other hand, the $\infty$-jet of the map $\phi_{c}$, in case of a $C^{\infty}$ vector field, is unique, so if there would exist an analytic central manifold then this last one is unique; in the foregoing example it is the $x$ axis. But for the (polynomial) example $\left(x-y^{2}\right) \partial / \partial x+y^{2} \partial / \partial y$ one can calculate that the $\infty$-jet of $x=\phi_{c}(y)$ is given by $j_{\infty} \phi_{c}(y)=\sum_{n \geq 1} n ! y^{n+1}$, which has a vanishing radius of convergence, so there is no analytic central manifold. On the other hand, by the Borel theorem we can choose a $C^{\infty}$ representative for $\phi_{c}$. This can be generalized in the planar case:

Proposition 1 If $n=2$ and if $X$ is $C^{\infty}$ and if the $\infty$-jet of $X$ in the direction of the central manifold is nonzero, then this central manifold is $C^{\infty}$. In particular, if $X$ is analytic then the central manifold is either an analytic curve of stationary points or is a $C^{\infty}$ curve along which $X$ has a nonzero jet.

See also [2] for proofs and additional reading. In general a central manifold is not necessarily $C^{\infty}$ (van Strien, 1979 and [1]): for the system in $\mathbf{R}^{3}$ given by $\left(x^{2}-z^{2}\right) \partial / \partial x+\left(y+x^{2}-z^{2}\right) \partial / \partial y+0 . \partial / \partial z$ one can find a $C^{k}$ central manifold for every $k$ but there is no $C^{\infty}$ central manifold. Indeed, in this case the domain of definition of $\phi_{c}$ shrinks to zero when $k$ tends to infinity.

\section{Central manifold reduction}

The importance of a central manifold lies in the principle of central manifold reduction, which roughly says that for local bifurcation phenomena it is enough 
to study the behavior on the central manifold, i.e. if two vector fields, restricted to their central manifolds, have homeomorphic integral curve portraits, and if the dimensions of $E_{s}$ and $E_{u}$ are equal, then the two vector fields have homeomorphic integral curve portraits in $\mathbf{R}^{n}$, at least locally near 0 . Let us become more precise.

Theorem 2 Let $m$ be the dimension of $E_{c}$. There exists $p, 0 \leq p \leq n-m$, such that $X$ is locally $C^{0}$ conjugate to

$$
X^{\prime}=\sum_{i=1}^{m} \tilde{X}_{i}\left(z_{1}, \ldots, z_{m}\right) \frac{\partial}{\partial z_{i}}+\sum_{i=m+1}^{m+p} z_{i} \frac{\partial}{\partial z_{i}}-\sum_{i=m+p+1}^{n} z_{i} \frac{\partial}{\partial z_{i}}
$$

where $\left(z_{1}, \ldots, z_{m}\right)$ is coordinate system on a central manifold, $\left(z_{1}, \ldots, z_{n}\right)$ is a coordinate system on $\mathbf{R}^{n}$ extending $\left(z_{1}, \ldots, z_{m}\right)$ and $\sum_{i=1}^{m} \tilde{X}_{i} \frac{\partial}{\partial z_{i}}$ is the restriction of $X$ to a central manifold. Moreover, if

$$
Y=\sum_{i=1}^{m} \tilde{Y}_{i}\left(z_{1}, \ldots, z_{m}\right) \frac{\partial}{\partial z_{i}}+\sum_{i=m+1}^{m+p} z_{i} \frac{\partial}{\partial z_{i}}-\sum_{i=m+p+1}^{n} z_{i} \frac{\partial}{\partial z_{i}}
$$

and if $\sum_{i=1}^{m} \tilde{Y}_{i} \frac{\partial}{\partial z_{i}}$ is $C^{0}$ equivalent (resp. $C^{0}$ conjugate) to $\sum_{i=1}^{m} \tilde{X}_{i} \frac{\partial}{\partial z_{i}}$ then $X$ is $C^{0}$ equivalent (resp. conjugate) to $Y$.

For a proof and further reading (a generalization) see [17].

In case that more smoothness than just $C^{0}$ is needed, we have the principle of normal linearization along the central manifold. More concretely, let $x$ denote a coordinate in the central manifold and let $y$ be a complementary variable, i.e. let $X=X_{c} \partial / \partial x+X_{h} \partial / \partial y$. We define the normally linear part along the central manifold by

$$
N X:=X_{c}(x, 0) \frac{\partial}{\partial x}+\frac{\partial X_{h}}{\partial y}(x, 0) \cdot y \frac{\partial}{\partial y}
$$


Under certain nonresonance conditions $[19,4]$ on the real parts of the eigenvalues of $d X(0)$ there exists a $C^{r}$ local conjugacy between $X$ and $N X$ for each $r \in \mathbf{N}$ (assuming $X$ of class $C^{\infty}$ ). If there are resonances, then one can conjugate with the so called seminormal or renormal form containing higher order terms, see $[5,4]$ and the references therein; here one can also find results in case that extra constraints should be respected, like symmetry, reversibility, invariance of some given foliation etcetera.

Parameters. Having an eigenvalue with zero real part is ungeneric, so in bifurcation problems we consider $p$-parameter families $X_{\lambda}$ near, say, $\lambda=0$. With respect to the results above we remark that such a family can be considered as a vector field near $(0,0) \in \mathbf{R}^{n} \times \mathbf{R}^{p}$ tangent to the leaves $\mathbf{R}^{n} \times\{\lambda\}$. In fact the parameter-direction $\mathbf{R}^{p}$ is contained in $E_{c}$. In all the mentioned results this structure 'of being a family' is respected. For example in theorem 2 we replace $\tilde{X}_{i}\left(z_{1}, \ldots, z_{m}\right)$ by $\tilde{X}_{i}\left(z_{1}, \ldots, z_{m}, \lambda\right)$. Hence, if $\tilde{X}_{\lambda}$ is a versal unfolding of $\tilde{X}_{0}$ then $X_{\lambda}$ is a versal unfolding of $X_{0}$. By this the search for versal unfoldings is reduced to the unfolding of singularities whose linear approximation at 0 has a purely imaginary spectrum.

Diffeomorphisms, periodic orbits. A completely analogous theory can be developed for fixed points of diffeomorphisms $f:\left(\mathbf{R}^{n}, 0\right) \rightarrow \mathbf{R}^{n}$. Here we split up the spectrum of the linear part $L=d f(0)$ at 0 as $\sigma(L)=\sigma_{s} \cup \sigma_{c} \cup \sigma_{u}$ where $\sigma_{s}$ resp. $\sigma_{c}$ resp. $\sigma_{u}$ consists of those eigenvalues with modulus $<1$ resp. $=1$ resp. $>1$. This theory can be applied to the time $t$-map of a vector field (and will give the same invariant manifolds) and to the Poincaré map of a 
transversal section of a periodic orbit of a vector field [9].

\section{Normal forms}

The general idea of a normal form is to put a (complicated) system into a form 'as simple as possible' by means of a change of coordinates. This idea was already developed to a great extent by H. Poincaré. Simple examples: 1) putting a square matrix into Jordan form, 2) the flow box theorem [1] near a nonsingular point. Depending on the context and on the purpose of the simplification, this concept may vary greatly. It depends on the kind of changes of coordinates that are tolerated (linear, polynomial, formal series, smooth, analytic) and on the possible structures that must be preserved (e.g. symplectic, volume-preserving, symmetric, reversible etcetera). Let us restrict to local normal forms, i.e. in the vicinity of a stationary point of a vector field or a diffeomorphism (the latter can be applied to the Poincaré map of a periodic orbit). We concentrate on the simplification of the Taylor series. The general idea is to apply consecutive polynomial changes of variables; at each step we simplify terms of a degree higher than in the step before. The ideal simplification would be to put all higher order terms to zero, which would (at least at the level of formal series) linearize the system. But as soon as there are resonances (see below), this is impossible: the planar system $2 x \partial / \partial x+\left(y+x^{2}\right) \partial / \partial y$ cannot be formally linearized.

Setting. Let $X$ be a $C^{r+1}$ vector field defined on a neighbourhood of $0 \in \mathbf{R}^{n}$, and denote $A=d X(0)$ (its linear approximation at 0 ). The Taylor 
expansion of $X$ at 0 takes the form

$$
X(x)=A \cdot x+\sum_{k=2}^{r} X_{k}(x)+O\left(|x|^{r+1}\right)
$$

where $X_{k} \in H^{k}$, the space of vector fields whose components are homogeneous polynomials of degree $k$. The classical formal normal form theorem is as follows. We define the operator $L_{A}$ on $H^{k}$ by putting $L_{A} h(x)=d h(x) \cdot A \cdot x-A \cdot h(x)$; one calls $L_{A}$ the homological operator. One checks that $L_{A}\left(H^{k}\right) \subset H^{k}$. One also denotes this by ad $A(h)(x)$ : see further in the Lie algebra setting. Let $R^{k}$ be the range of $L_{A}$, i.e. $R^{k}=L_{A}\left(H^{k}\right)$. Let $G^{k}$ denote any complementary subspace to $R^{k}$ in $H^{k}$. The formal normal form theorem states, under the above settings:

Theorem 3 [9, 11] There exists a composition of near identity changes of variables of the form

$$
x=y+\xi^{k}(y)
$$

where the components of $\xi^{k}$ are homogeneous polynomials of degree $k$, such that the vector field $X$ is transformed into

$$
Y(y)=A . y+\sum_{k=2}^{r} g_{k}(y)+O\left(|y|^{r+1}\right)
$$

where $g_{k} \in G^{k}, k=2, \ldots, r$.

Sometimes this theorem is applied to the restriction of a vector field to its central manifold, for reasons explained in section 3. This is the reason why we did not assume $X$ to be $C^{\infty}$; in the latter case one can let $r \rightarrow \infty$ and obtain a normal form on the level of formal Taylor series (also called $\infty$-jets). Using a theorem of Borel we infer the existence of a $C^{\infty}$ change of variables $\phi$ such 
that the Taylor series of $\phi_{*}(X)$ is $A . y+\sum_{k=2}^{\infty} g_{k}(y)$. For practical computations it is often appropriate to first simplify the linear part $A$ and to diagonalize it whenever possible. Hence it is convenient to use a complexified setting and to use complex polynomials or power series. One can show that all involved changes of variables preserve the property of 'being a complex system coming from a real system', i.e. at the final stage we can return to a real system. See e.g. [1] for a more precise mathematical description.

Hence we can assume that $A$ is an upper triangular matrix. Let the eigenvalues be $\lambda_{1}, \ldots, \lambda_{n}$. It can be calculated that the eigenvalues of $L_{A}$, as an operator $H^{k} \rightarrow H^{k}$, are then the numbers $\langle\lambda, \alpha\rangle-\lambda_{j}$ where $\alpha \in \mathbf{N}^{n}, \sum_{j=1}^{n} \alpha_{j}=k$ and $1 \leq j \leq n$. Hence, if these would all be nonzero then $B^{k}=H^{k}$ and then we have an ideal simplification i.e. all $g_{k}$ equal to zero. However, if such a number is zero, that is,

$$
\langle\lambda, \alpha\rangle-\lambda_{j}=0
$$

this is called a resonance between the eigenvalues. In such a case we have to choose a complementary space $G^{k}$. From linear algebra it follows that one can always choose

$$
G^{k}=\operatorname{ker}\left(L_{A^{*}}\right)
$$

where $A^{*}$ is the adjoint operator. But this choice (3) is not unique and is from the computational point of view not always optimal, especially if there are nilpotent blocks. This fact has been exploited by many authors. A typical example for this is the case where $A=y \partial / \partial x$. On the other hand if $A$ is semisimple we can choose the complementary space to be $\operatorname{ker}\left(L_{A}\right)$, so $L_{A} g_{k}=0$; 
we can assume it to be the (complex) diagonal $\left[\lambda_{1}, \ldots, \lambda_{n}\right]$. In that case we can be more explicit as follows. Let $e_{j}=\partial / \partial x_{j}$ denote the standard basis on $\mathbf{C}^{n}$. For a monomial one can calculate that

$$
L_{A}\left(x^{\alpha} e_{j}\right)=\left(\langle\lambda, \alpha\rangle-\lambda_{j}\right) x^{\alpha} e_{j}
$$

If the latter is zero, then the monomial is called resonant. This implies that the normal form can be chosen so that it only contains resonant monomials.

Putting a system into normal form not only simplifies the original system, it also gives more geometric insight on the Taylor series. To be more precise, suppose (for simplicity: this can be generalized [11]) that $A$ is semisimple. One can calculate that the condition $L_{A} g_{k}=0$ implies: $\exp (-A t) g_{k}(\exp (A t) x)=$ $g_{k}(x)$ for all $t \in \mathbf{R}$. This means that $g_{k}$ is invariant for the 1-parameter group $\exp (A t)$. A typical example in the plane is: $A$ has eigenvalues $i \lambda,-i \lambda$. Note that the (only) resonances are $\langle(i \lambda,-i \lambda),(p+1, p)\rangle-i \lambda=0$ and $\langle(i \lambda,-i \lambda),(p, p+1)\rangle+$ $i \lambda=0$ for all $p \in \mathbf{N}$. We suppose that the original system was real i.e. on $\mathbf{R}^{2}$; we can choose linear coordinates such that for $z=x+i y, \bar{z}=x-i y$ the linear part is $A=\operatorname{diagonal}[i \lambda,-i \lambda]$. Applying the remarks above we conclude that the normal form only contains the monomials $(z \bar{z})^{p} z \partial / \partial z$ and $(z \bar{z})^{p} \bar{z} \partial / \partial \bar{z}$. The geometric interpretation here is that these monomials are invariant for rotations around $(0,0)$. This can also be seen on the real variant of this: the Taylor series of the (real) normalized system has the form $\left(\lambda+f\left(x^{2}+y^{2}\right)\right)(x \partial / \partial y-$ $y \partial / \partial x)+g\left(x^{2}+y^{2}\right)(x \partial / \partial x+y \partial / \partial y)$ and is invariant for rotations. Warning: the dynamic behaviour of a formal normal form in the central manifold can be 
very different from that of the original vector field, since we are only looking at the formal level. A trivial example is (take $f=g=0$ in the foregoing example) $X(x, y)=\lambda(x \partial y-y \partial x)-\exp \left(-1 /\left(x^{2}\right)\right) \partial / \partial x$, where orbits near $(0,0)$ spiral to $(0,0)$, whereas the normal form is just a linear rotation. This difference is due to the so called flat terms, i.e. the difference between the transformed vector field and a $C^{\infty}$ realization of its normalized Taylor series (or polynomial). In case of analyticity of $X$ one can ask for analyticity of the normalizing transformation $\phi$. Generically this is not the case in many situations. The precise meaning of this 'genericity condition' is too elaborate to explain in this repository paper. We provide some further reading in section 5 . One could roughly say that, in the central manifold, the normal form has too much symmetries and is too poor to model more complicated dynamics of the system, which can be 'hidden in the flat terms'. To quote Il'yashenko [13]: 'In the theory of normal forms of analytic differential equations, divergence is the rule and convergence the exception ...'

In many applications we want to preserve some extra structure, such as a symplectic structure, a volume form, some symmetry, reversibility, some projection etcetera; the case of a projection is important since it includes vector fields depending on a parameter. Sometimes a superposition of these structures appears (e.g. a family of volume preserving systems). We would like that the normal form procedure respects this structure at each step. One can often formulate this in terms of vector fields belonging to some Lie subalgebra $\mathcal{L}_{0}$. The idea is then to use changes of variables like (1) where $\xi_{k}$ is then generated by a vector field in $\mathcal{L}_{0}$. This will guarantee that all changes of variables are 
'compatible' with the extra structure. Unlike the general case where we could work with monomials like in (4) we will have to consider vector fields $h_{k}$ in $\mathcal{L}_{0}$ whose components are homogeneous polynomials of degree $k$. If this can be done, one says that $\mathcal{L}_{0}$ respects the grading by the homogeneous polynomials. In order to fix the ideas: suppose that $\mathcal{L}_{0}$ are the divergence free planar vector fields. Remark that a monomial $x^{i} y^{j} \partial / \partial x$ is not divergence free. We can instead use time one mappings of homogeneous vector fields of the form $a(q+1) x^{p+1} y^{q} \partial / \partial x-a(p+1) x^{p} y^{q+1} \partial / \partial y$. Up to terms of higher order we can use the time one of $h_{k}$ instead of $x+h_{k}(x)$. In case that one asks for a $C^{\infty}$ realization of the normalizing transformation, we need an extra assumption on the extra structure, i.e. on $\mathcal{L}_{0}$, called the Borel property: denote $J_{\infty, 0}$ the set of formal series such that each truncation is the Taylor polynomial of an element of $\mathcal{L}_{0}$. The extra assumption is: each element of $J_{\infty, 0}$ must be the Taylor series of a $C^{\infty}$ vector field in $\mathcal{L}_{0}$. It can be proved [6] that the following structures respect the grading and satisfy the Borel property: being an $r$-parameter family, respecting a volume form on $\mathbf{R}^{n}$, being a Hamiltonian vector field ( $n$ even), being reversible for a linear involution.

One could consider other types of grading of the involved Lie-algebras.

This method, using the framework of the so called filtered Lie algebras, is explained and developed systematically in a more general and abstract context in $[6]$.

In nonlocal bifurcations, such as near a homoclinic loop for example, it is not enough to perform central manifold reduction near the singularity: a simplified 
smooth model in a full neighbourhood of the singularity is often needed, for example in order to compute Poincaré maps.

Let us start with the 'purely' hyperbolic case (i.e. $\operatorname{dim} E_{c}=0$ ). First we compute the formal normal form like above. If there are no resonances (2) then we can formally linearize the vector field $X$. If $X$ is $C^{\infty}$ then a classical theorem of Sternberg (1958) states that this linearization can be realized by a $C^{\infty}$ change of variables (i.e. no more flat terms remaining). In case there are resonances, we must allow nonlinear terms: the resonant monomials. Also in this case we can reduce $C^{\infty}$ to this normal form. Using the same methods it is also possible to reduce to a polynomial normal form, but this time using $C^{k}(k<\infty)$ changes of variables. More precisely, if $k$ is a given number and if we write the vector field as $X=X_{N}+R_{N}$ where $X_{N}$ is the Taylor polynomial up to order $N$ (which can be assumed to be in normal form) and where $R_{N}(x)=O\left(|x|^{N+1}\right)$, then for $N$ sufficiently large there is a $C^{k}$ change of variables conjugating $X$ to $X_{N}$ near 0 . The number $N$ depends on the spectrum of $A=d X(0)$. An elegant proof of these facts can be found in [15]. In case that extra structure must be preserved: see [4], which also deals with the partially hyperbolic case ( $\left.\operatorname{dim} E_{c} \geq 1\right)$. As already remarked above, the case of a parameter-dependent family can be regarded as a partially hyperbolic stationary point preserving this extra structure.

The question of an analytic normal form, also in the hyperbolic case, leads to convergence questions and calls upon so called small divisor problems. The classical results are due to Poincaré and Siegel; let us summarize them; they are 
formulated in the complex analytic setting:

Theorem 4 (a) If the convex hull of the spectrum of $A$ does not contain $0 \in \mathbf{C}$ then $X$ can locally be put into normal form by an analytic change of variables. Moreover this normal form is polynomial.

(b) If the spectrum $\left\{\lambda_{1}, \ldots, \lambda_{n}\right\}$ of $A$ satisfies the condition that there exists $C>0$ and $\mu>0$ such that for any $m \in \mathbf{N}^{n}$ with $\sum_{j} m_{j} \geq 2$ :

$$
\left|\left\langle\left(\lambda_{1}, \ldots, \lambda_{n}\right), m\right\rangle-\lambda_{j}\right| \geq \frac{C}{|m|^{\mu}}
$$

for $1 \leq j \leq n$ then $X$ can be locally linearized by an analytic change of variables.

Note that case (a) contains the case where 0 is a hyperbolic source or sink. This case (a) in theorem 4 can be extended if there are parameters: if $X$ depends analytically on a parameter $\varepsilon \in \mathbf{C}^{p}$ near $\varepsilon=0$ then the change of variables is also analytic in $\varepsilon$; moreover the normal form is then a polynomial in the space variables whose coefficients are analytically dependent on the parameter $\varepsilon$.

For case (b) this is surely not the case, since the condition (5) is fragile: a small distortion of the parameter generically causes resonances, be it of a high order. To fix the ideas: consider $n=2$ and suppose $\lambda_{1}<0<\lambda_{2}$. By a generic but arbitrary small perturbation we can have that the ratio of these eigenvalues becomes a negative rational number $-p / q$, which gives a resonance of the form (2) with $j=1$ and $\alpha=(q+1, p)$, so (5) is violated.

So analytic linearization, or even a polynomial analytic normal form, is ungeneric for families of such hyperbolic stationary points. The search for analytic normal forms, i.e. simplified models, for families is still under investigation. A 
first simplification is obtained via the stable and unstable manifold from theorem 1, that is: the graphs of $\phi_{s s}$ and $\phi_{u u}$. When $X$ is analytic near 0 then these manifolds are also analytic. So up to an analytic change of variables we can assume that $E_{s}$ and $E_{u}$ are invariant, which gives a simplification of the expression of $X$. Moreover there is analytic dependence on parameters.

For local diffeomorphisms there are completely similar theorems about all the above.

\section{$5 \quad$ Further reading}

The concept of central manifold can be extended to more general invariant sets: see $[10]$ and the references in that paper. It can also be extended to the infinite dimensional case and can be applied to partial differential equations: see [21].

Concerning the generic divergence of normalizing transformations: $[7,8,13$, 14]. Although the power series giving the normalizing transformation generally diverges, the study of the dynamics is often performed by truncating the normal form at a certain order. Recently, in [16] the question about what is an optimal truncation is studied. It is shown, in case $d X(0)$ is semi-simple, that the order of the normal form can be optimized so that the remainder satisfies some estimate shrinking exponentially fast to zero as a function of the radius of the domain.

Concerning normal forms preserving the Hamiltonian structure see $[3,18]$ for a starting point; this is an extended subject on its own, sometimes called Birkhoff normal form, and it would require another repository paper. 
Further simplifications of the normal form can sometimes be obtained by taking into account nonlinear terms (instead of just $A$ ) in order to obtain reductions of higher order terms: see [12] and especially the reference list therein.

More further reading about applications of normal forms and central manifolds to bifurcation theory: [11].

\section{References}

[1] D. Arrowsmith and C. Place. Dynamical Systems. Cambridge University Press, 1990.

[2] B. Aulbach. One-dimensional center manifolds are $C^{\infty}$. Results in Mathematics, 21:3-11, 1992.

[3] G. D. Birkhoff. Dynamical systems. With an addendum by Jurgen Moser. American Mathematical Society Colloquium Publications, Vol. IX. American Mathematical Society, Providence, R.I., 1966.

[4] P. Bonckaert. Conjugacy of vector fields respecting additional properties. Journal of Dynamical and Control Systems, 3:419-432, 1997.

[5] P. Bonckaert. Symmetric and reversible families of vector fields near a partially hyperbolic singularity. Ergodic Theory and Dynamical Systems, 20:1627-1638, 2000.

[6] H. Broer. Formal normal forms for vector fields and some consequences for bifurcations in the volume preserving case. In Dynamical Systems and 
Turbulence, Warwick 1980, volume 898 of Lecture Notes in Mathematics. Springer-Verlag, 1981.

[7] H. Broer and F. Takens. Formally symmetric normal forms and genericity. Dynamics Reported Ser. Dynam. Systems Appl., 2:11-18, 1989.

[8] A. D. Bruno. Local methods in Nonlinear Differential Equations. SpringerVerlag, New York, 1989.

[9] S.-N. Chow, C. Li, and D. Wang. Normal forms and bifurcations of planar vector fields. Cambridge University Press, 1994.

[10] S.-N. Chow, W. Liu, and Y. Yi. Center manifolds for invariant sets. Journal of Differential Equations, 168:355-385, 2000.

[11] F. Dumortier. Local study of planar vector fields: singularities and their unfoldings. In Structures in Dynamics, volume 2 of Studies in Mathematical Physics, pages 161-241. Elsevier, 1991.

[12] G. Gaeta. Poincaré normal and renormalized forms. Acta Appl. Math., 70(1-3):113-131, 2002. Symmetry and perturbation theory.

[13] Y. S. Il'yashenko. In the theory of normal forms of analytic differential equations violating the conditions of Bryuno divergence is the rule and convergence the exception. Moscow Univ. Math. Bull, 36(2):11-18, 1981.

[14] Y. S. Il'yashenko and A. S. Pyartli. Materialization of resonances and divergence of normalizing series for polynomial differential equations. Journal of Mathematical Sciences, 32 (3):300-313, 1986. 
[15] Y. S. Il'yashenko and S. Y. Yakovenko. Finitely smooth normal forms of local families of diffeomorphisms and vector fields. Russian Math. Surveys, 46:1-43, 1991 .

[16] G. Iooss and E. Lombardi. Polynomial normal forms with exponentially small remainder for analytic vector fields. Journal of Differential Equations, 212:1-61, 2005 .

[17] J. Palis and F. Takens. Topological equivalence of normally hyperbolic dynamical systems. Topology, 16(4):335-345, 1977.

[18] C. L. Siegel and J. K. Moser. Lectures on celestial mechanics. Springer, Berlin, 1971, reprint 1995.

[19] F. Takens. Partially hyperbolic fixed points. Topology, 10:133-147, 1971.

[20] A. Vanderbauwhede. Center manifolds, normal forms and elementary bifurcations. In U. Kirchgraber and O. Walther, editors, Dynamics Reported, volume 2, pages 89-169. Wiley, New York, 1989.

[21] A. Vanderbauwhede and G. Iooss. Center manifold theory in infinite dimensions. In C. J. et al., editor, Dynamics Reported, volume 1 New Series, pages 125-163. Springer-Verlag, 1992. 